Article

\title{
Optimal Scheduling and Real-Time State-of-Charge Management of Energy Storage System for Frequency Regulation
}

\author{
Jin-Sun Yang ${ }^{1}$, Jin-Young Choi ${ }^{1}$, Geon-Ho An ${ }^{2}$, Young-Jun Choi ${ }^{2}$, Myoung-Hoe Kim ${ }^{2}$ and \\ Dong-Jun Won ${ }^{1, *}$ \\ 1 Department of Electrical Engineering, Inha University, Incheon 22212, Korea; sealoveyjs@gmail.com (J.-S.Y.); \\ jy128308@gmail.com (J.-Y.C.) \\ 2 Department of Power Grid Integration of Research and Development (R\&D) Center, Hyosung Corporation, \\ Anyang 14080, Korea; geonhoan@hyosung.com (G.-H.A.); swot87@hyosung.com (Y.-J.C.); \\ runner@hyosung.com (M.-H.K.) \\ * Correspondence: djwon@inha.ac.kr or djwon777@gmail.com; Tel.: +82-32-860-7404
}

Academic Editor: William Holderbaum

Received: 9 August 2016; Accepted: 22 November 2016; Published: 30 November 2016

\begin{abstract}
An energy storage system (ESS) in a power system facilitates tasks such as renewable integration, peak shaving, and the use of ancillary services. Among the various functions of an ESS, this study focused on frequency regulation (or secondary reserve). This paper presents an optimal scheduling algorithm for frequency regulation by an ESS. This algorithm determines the bidding capacity and base point of an ESS in each operational period to achieve the maximum profit within a stable state-of-charge $(S O C)$ range. However, the charging/discharging efficiency of an ESS causes SOC errors whenever the ESS performs frequency regulation. With an increase in SOC errors, the ESS cannot respond to an automatic generation control (AGC) signal. This situation results in low ESS performance scores, and finally, the ESS is disqualified from performing frequency regulation. This paper also presents a real-time $S O C$ management algorithm aimed at solving the $S O C$ error problem in real-time operations. This algorithm compensates for $S O C$ errors by changing the base point of the ESS. The optimal scheduling algorithm is implemented in MATLAB by using the particle swarm optimization (PSO) method. In addition, changes in the SOC when the ESS performs frequency regulation in a real-time operation are confirmed using the PSCAD/EMTDC tool. The simulation results show that the optimal scheduling algorithm manages the SOC more efficiently than a commonly employed planning method. In addition, the proposed real-time SOC management algorithm is confirmed to be capable of performing $S O C$ recovery.
\end{abstract}

Keywords: energy storage system (ESS); frequency regulation (FR); optimal scheduling; state-ofcharge (SOC); energy management

\section{Introduction}

Recent power systems are focusing on energy storage systems (ESSs) because of their ability to store energy. Many studies have examined ESSs to utilize renewable integration, peak shaving, ancillary services, and microgrids [1-10]. In [11], the authors presented the feasibility of an ESS for the operation of an island AC microgrid with photovoltaic generation. In [12], the authors proposed an ESS management algorithm for full renewable energy source (RES) exploitation. This algorithm was shown to minimize the curtailment of RES energy generation through energy buffering and forecasting error compensation by an ESS. A one-day-ahead scheduling procedure was combined with a real-time control strategy to improve RES generation and reliability. This study shows that excellent forecasting error compensation can be achieved even with ESSs of moderate size. In recent years, 
many studies have focused on the ability of ESSs to enable frequency regulation, given that its ramp rate is higher than that of conventional resources. In [13], the authors proposed an alternative market structure with the ability to efficiently demand response (DR) and automatic generation control (AGC) and accommodate the intermittency and uncertainty that are concomitant in renewable generation, thereby leading to the efficient integration of renewables at the market level and reduced regulation requirements at the AGC level. This paper proposed an integrated dynamic market mechanism (DMM) that combined a real-time market clearing procedure with AGC. DMM implementation enabled more frequent economic dispatch than the optimal power flow (OPF). Our proposed algorithm also reduces the root mean square error of the area control error by using an aggregate frequency error. In [14], the authors proposed a hybrid operation strategy for a wind energy conversion system with a battery energy storage system (BESS) to support frequency control. In their study, the output power command of the BESS was determined according to three factors: the state-of-charge (SOC), frequency deviation, and load variation. Their proposed operation strategy significantly improved the initial low-frequency response and provided a superior contribution to short-term frequency regulation. The research of [15] proposed an SOC feedback control scheme and investigated the performance of the grid frequency deviation response. The control was integrated with the widely used wind turbine blade pitch control and the speed governor control of a local synchronous generator. It was shown that the ESS helped supply power to wind farms to support frequency regulation and effectively regulated the battery SOC. An outcome of the increasing number of studies conducted on ESS frequency control is the proposal of a novel approach associated with the actual performance measurement of an ESS conducting frequency regulation [16]. This paper evaluated the current methods for procuring, dispatching, and compensating resources for frequency regulation. The authors also calculated the performance payment of the proposed novel approach by employing a sigmoid function. This approach influenced revenue by adjusting the calculation of the performance score. This approach will provide further insight into administrative price adjustment. The operator would be able to estimate the total mileage expected on an annual cost, and they would be able to calculate an administrative price that would reflect a fair compensation for the resources based on their actual frequency-regulation contribution and performance. In [17], the authors proposed a model that decided the optimal joint bidding strategy of battery storage in joint day-ahead energy, reserve, and regulation markets. Their novel algorithm considering the battery life cycle significantly improved a storage battery's overall economics in performance-based regulation.

At present, an ESS participates in the frequency regulation market by bidding its maximum capacity. However, an ESS is unable to manage its SOC because the power system operator sends an AGC signal without considering the SOC of an ESS. If an ESS is unable to manage its SOC, it does not respond to the AGC signal; therefore, an ESS should be able to manage its SOC. Many studies have focused on SOC management via day-ahead scheduling or via compensation by renewable generation; however, management by these methods in a real-time operation is difficult.

This study proposes an optimal scheduling algorithm and a real-time SOC management algorithm for frequency regulation by an ESS. The optimal scheduling algorithm determines the bidding capacity and base point through the particle swarm optimization (PSO) method for achieving the maximum profit through SOC management. Further, the real-time SOC management algorithm recovers the $S O C$ in a real-time operation via participation in the energy market. This algorithm manages the SOC of an ESS by rebidding the scheduled base point in the energy market. Furthermore, a simulation is performed using actual PJM (Monroe, MN, USA) operation data. This paper is organized as follows. Section 2 explains the frequency regulation service and frequency regulation market. Section 3 presents the optimal scheduling algorithm and compares it with a commonly employed planning method. Section 4 presents the proposed real-time SOC management algorithm. Section 5 presents simulation cases and discusses the simulation results. Section 6 summarizes the conclusions and suggests future work. 


\section{Frequency Regulation Market}

In a power system, the frequency changes continuously because of an imbalance of supply and demand. The system operators conduct frequency regulation or secondary reserve to reduce the fluctuations and thereby provide stable and reliable system operation. Frequency regulation involves the injection or withdrawal of as much active power as the assigned regulation capacity of the resource [18]. Two types of frequency regulation market exist. The first frequency regulation market type is separated into regulation up and regulation down. Examples include New York Independent System Operator (NYISO, Rensselaer, NY, USA) and California Independent System Operator (CAISO, Folsom, CA, USA), and ISO of Europe. The second frequency regulation market type does not separate regulation up and regulation down and examples for this type include PJM and Midcontinent Independent System Operator (MISO, Carmel, CA, USA). The second frequency regulation market type was assumed in this study because PJM data were used; the frequency regulation market in PJM was selected as the test bed, and simulation was performed according to PJM frequency regulation market rules. To provide frequency regulation, resources bid on the frequency regulation market according to the requisite capacity in each operation time [19]. The resources provide as much active power as the AGC signal whenever the resources receive an AGC signal; this output point is called the "set point". If the resources participate in the energy market, the resources provide as much active power as the bidding quantity every time; this output point is called the "base point". The resources participating in both frequency regulation and the energy market provide as much active power as the base point and perform frequency regulation whenever the resources receive an AGC signal by additionally providing as much active power as the set point [20]. Figure 1 illustrates the concept of the set point and base point of an ESS.

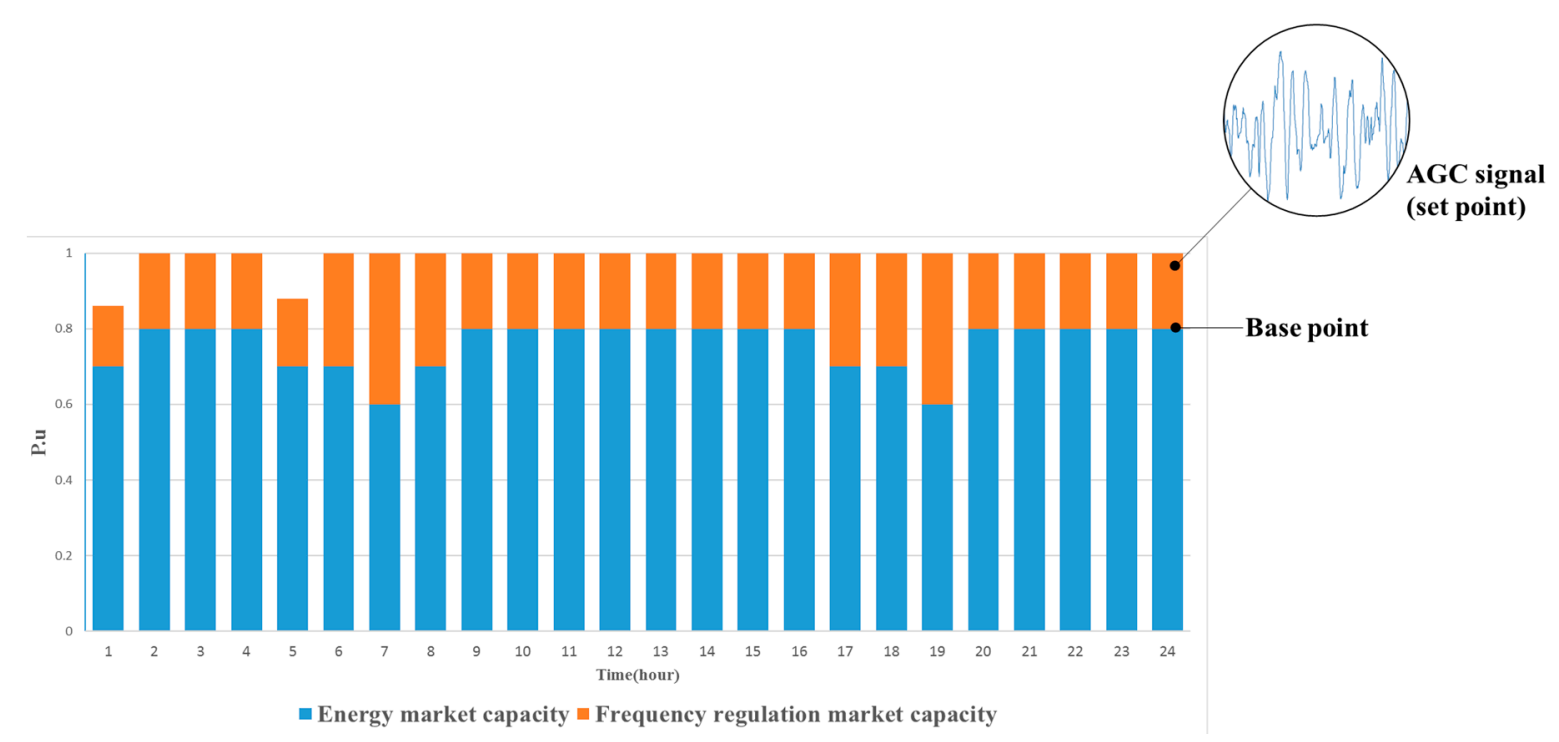

Figure 1. The concept of set point and base point. AGC: automatic generation control.

An ESS always provides active power according to the base point with the assigned energy and provides active power according to the set point when the ESS receives an AGC signal. Therefore, an ESS provides as much active power as the output point. The data used in this paper consist of the actual operation data of the PJM region. The hourly averaged AGC signal, regulation market capacity clearing price, regulation market performance clearing price, and locational marginal price were used to plan the optimal scheduling [21-23]. A real-time operation simulation was performed using an actual AGC signal. The data were collected on 5 September 2013. Figure 2 shows the hourly market price data, hourly averaged AGC signal, and actual AGC signal. 


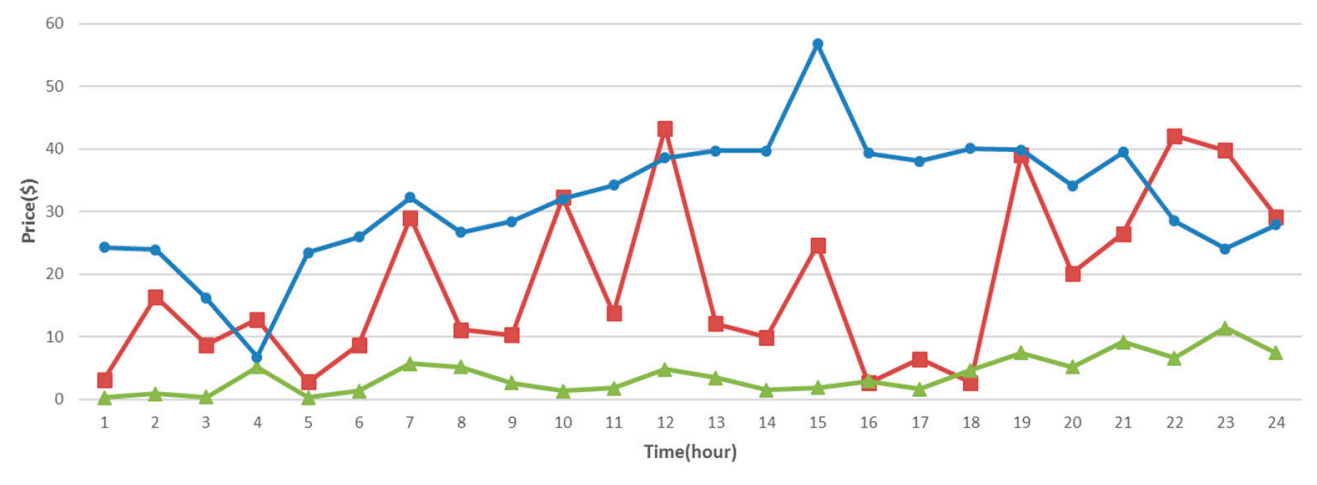

(a)

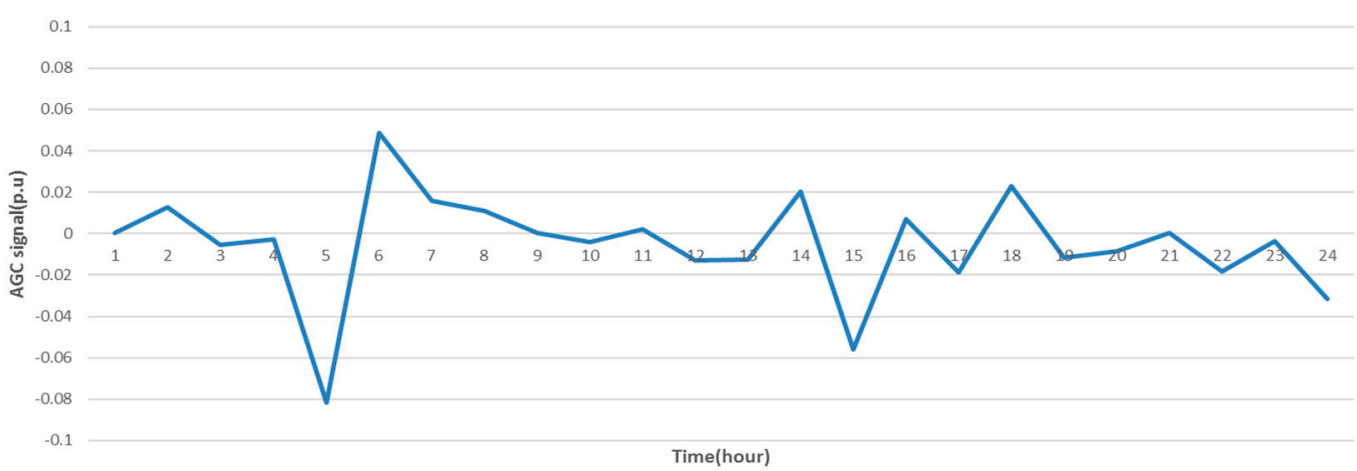

(b)

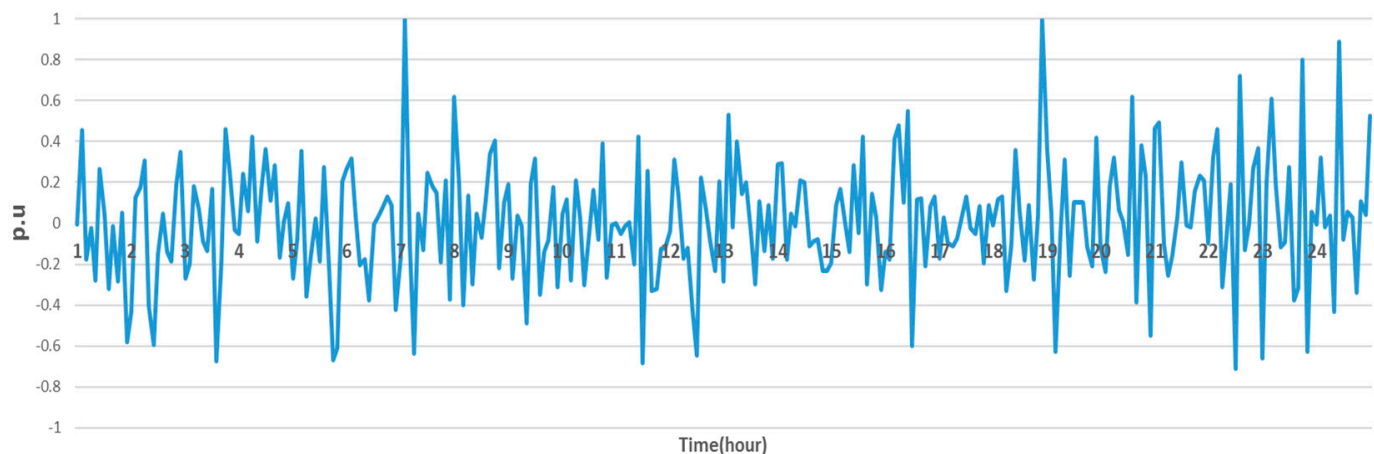

(c)

Figure 2. Actual market data in PJM (a) hourly market price; (b) hourly averaged AGC signal; and (c) AGC signal. RMCCP: regulation market capability clearing price; $R M P C P$ : regulation market performance clearing price; and $L M P$ : locational marginal pricing.

\section{Optimal Scheduling Algorithm}

To provide frequency regulation, ESS owners bid the maximum capacity in the day-ahead market, but they do not manage the $S O C$ in the same manner. The optimal scheduling algorithm schedules the bidding plan of an ESS to earn the maximum profit within a stable $S O C$ range. When a schedule is determined, ESS owners bid on the frequency regulation market in day-ahead market according to the schedule. For a scheduling plan, this study chose $4 \mathrm{MW} / 2 \mathrm{MWh}$ Li-ion batteries as the ESS and set the charging/discharging efficiency to $91 \%$. The initial SOC was set at $60 \%$. This section compares the $S O C$, frequency regulation profit, and scheduling results of the maximum-capacity bidding plan and the optimal scheduling algorithm. 


\subsection{Maximum-Capacity Bidding Plan}

The maximum-capacity bidding plan is a conventional bidding method in the frequency regulation market. In this plan, the base point is set to zero. Therefore, the bidding capacity is $4 \mathrm{MW}$, and the base point is set to 0 p.u. The $S O C$ changes are calculated as follows Equation (1):

$$
\begin{aligned}
& \text { If } \overline{A G C}(i) \leq 0, \operatorname{SOC}(i+1)=\operatorname{SOC}(i)-\frac{c_{\text {bid }} \times \overline{A G C}(i)}{C_{\text {rated }}} \times \eta_{c} \times \Delta t \\
& \text { If } \overline{A G C}(i)>0, \operatorname{SOC}(i+1)=\operatorname{SOC}(i)-\frac{c_{b i d} \times \overline{A G C}(i)}{C_{\text {rated }}} \times \frac{1}{\eta_{d}} \times \Delta t
\end{aligned}
$$

where $i$ is the index of time (h), SOC (i) is the SOC of the ESS at time $i, c_{b i d}$ is the bidding capacity of ESS, $C_{\text {rated }}$ is the rated capacity of ESS, $\overline{A G C}(i)$ is the hourly averaged AGC signal, and $\eta_{c}$ and $\eta_{d}$ are the charging and discharging efficiencies of the ESS, respectively. Figure 3 presents the bidding capacity, base point, and SOC when the ESS is bid by the maximum-capacity bidding plan. In addition, Table 1 lists the frequency regulation profit.

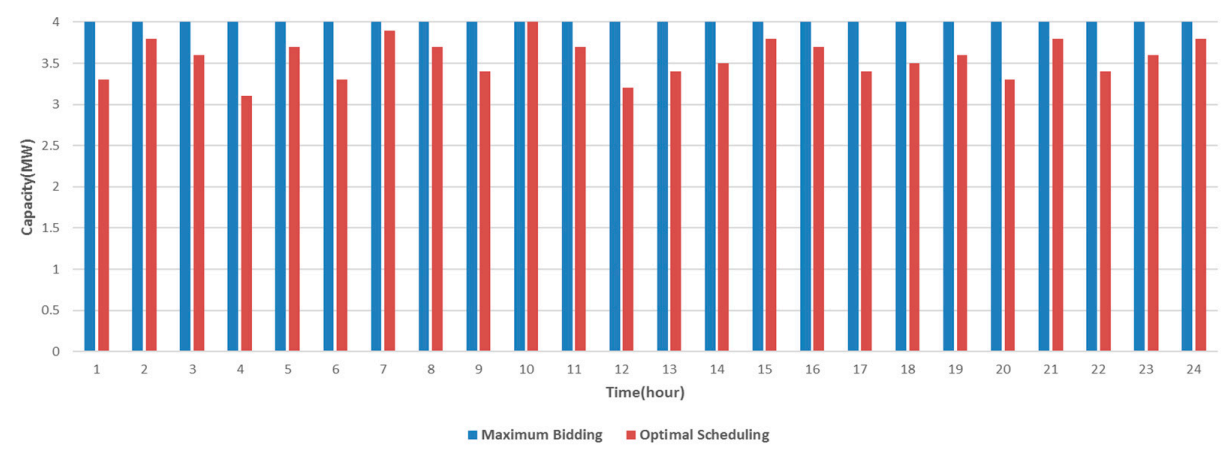

(a)

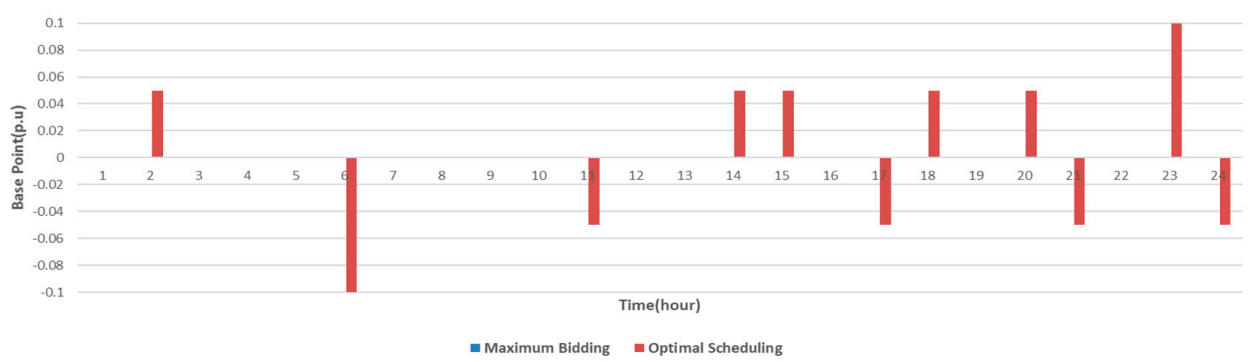

(b)

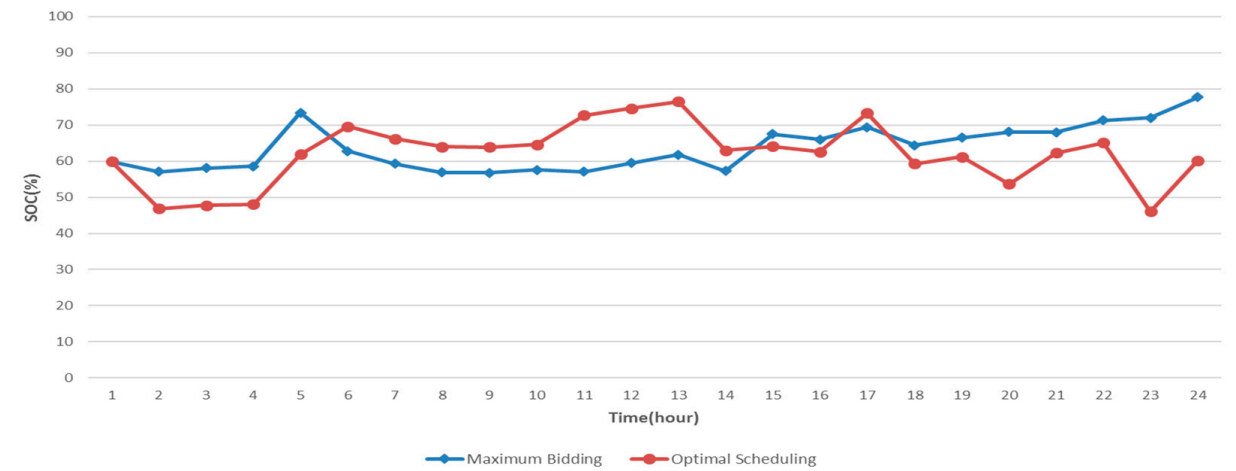

(c)

Figure 3. Maximum capacity bidding plan and optimal scheduling algorithm results (a) bidding capacity; (b) base point; and (c) SOC. 
Table 1. Frequency regulation scheduling profits.

\begin{tabular}{cccc}
\hline Method & Regulation Profit & Energy Profit & Total Profit \\
\hline Maximum capacity bidding plan & $\$ 2773.4$ & $\$ 0$ & $\$ 2773.4$ \\
Optimal scheduling algorithm & $\$ 2483.1$ & $\$ 9.4$ & $\$ 2492.5$ \\
\hline
\end{tabular}

\subsection{Optimal Scheduling Algorithm}

The optimal-scheduling algorithm is a method used to earn the maximum profit within a stable SOC using the PSO method. PSO is a widely used traditional method for solving problems in power systems [24-27]. PSO solves the optimization problem as follows:

$$
\begin{gathered}
x_{k+1}^{j}=x_{k}^{j}+v_{k+1}^{j} \\
v_{k+1}^{j}=w v_{k}^{j}+c_{1} r_{1}\left(P_{k}^{j}-x_{k}^{j}\right)+c_{2} r_{2}\left(P_{k}^{g}-x_{k}^{j}\right)
\end{gathered}
$$

where $P_{k}^{j}$ and $P_{k}^{g}$ are the individual best position and the global best position, respectively; $k$ and $j$ represent the iteration number and particle number, respectively; $x$ is the position of the particle; $v$ is the velocity; $w$ is the inertia weight; $c_{1}$ and $c_{2}$ are acceleration coefficients, and $r_{1}$ and $r_{2}$ are randomly generated numbers in the range of $[0,1]$. The PSO method finds a solution to minimize the cost function, $F\left(x_{i}\right)$. To minimize the cost function $F\left(x_{i}\right)$ in particle $j$, PSO calculates the individual best position of particle $j$ using each $x_{i}$ and updates each $x_{i}$ position as much as the velocities to find the individual best position in each iteration $k$. Finally, PSO is used to compare the individual best position to update the global best position and determine a solution.

To plan the optimal scheduling, the cost function, $F\left(x_{i}\right)$, was formulated as:

$$
\begin{gathered}
\max \sum_{i=1}^{24} C_{o}(i) \\
C_{o}(i)=C_{c}(i)+C_{p}(i)-C_{e}(i) \\
C_{c}(i)=c\left(x_{i}\right) \times R M C C P(i) \times P S \\
C_{p}(i)=c\left(x_{i}\right) \times R M P C P(i) \times P S \times M R \\
C_{e}(i)=c\left(x_{i}\right) \times \operatorname{LMP}(i) \times b p\left(x_{i}\right)
\end{gathered}
$$

subject to the following constraints:

$$
\begin{gathered}
\operatorname{SOC}(0)=S O C(24) \\
S O C_{\min } \leq S O C(i) \leq S O C_{\max } \\
\text { If } \overline{A G C}(i) \leq 0, S O C(i+1)=S O C(i)-\frac{c_{b i d} \times\left[\overline{A G C}(i)+b p\left(x_{i}\right)\right]}{C_{\text {rated }}} \times \eta_{c} \times \Delta t \\
\text { If } \overline{A G C}(i)>0, S O C(i+1)=S O C(i)-\frac{c_{b i d} \times\left[\overline{A G C}(i)+b p\left(x_{i}\right)\right]}{C_{\text {rated }}} \times \frac{1}{\eta_{d}} \times \Delta t \\
c\left(x_{i}\right)+c\left(x_{i}\right) \times b p\left(x_{i}\right) \leq \text { Maximum capacity }
\end{gathered}
$$

where $C_{o}(i)$ is the operation profit, $C_{c}(i)$ and $C_{p}(i)$ are the regulation capability credit and regulation performance credit, respectively; $C_{e}(i)$ is the cost by bidding in the energy market; $c\left(x_{i}\right)$ and $b p\left(x_{i}\right)$ are the scheduled bidding capacity and base point in the energy market by PSO, respectively; RMCCP (i), RMPCP (i), and LMP (i) are the hourly regulation market capability clearing price, the hourly regulation market performance clearing price, and the hourly locational marginal pricing, respectively. $P S$ and $M R$ are the performance score and mileage ratio, respectively. Note that the initial SOC and final SOC are considered in constraint Equation (9). For operation within a stable 
$S O C$ range, the $S O C$ management constraint is set as in Equation (10). The $S O C$ was calculated using constraint Equation (11). Constraint Equation (12) manages the bidding capacity up to the maximum capacity. For the optimal scheduling algorithm, the upper and lower limits of the stable SOC range, $S_{\min }$ and $S_{\max }$, are set to $40 \%$ and $80 \%$, respectively. Typically, ESSs achieve a performance score of 0.95; thus, $P S$ was assumed to be 0.95 . In addition, the MR on 5 September 2013 was 3 . To perform optimal scheduling, the data described in Section 3 were used, and Equations (4)-(12) were solved in MATLAB/Simulink (MathWorks, Natick, MA, USA). Figure 3 shows the changes in the bidding capacity, base point, and SOC. Table 1 lists the frequency regulation profits. Regulation profit is the benefit participating frequency regulation market, and energy profit is the cost participating energy market. As shown in Table 1, the maximum-capacity bidding plan earned higher frequency regulation profit than that of the optimal scheduling algorithm. On the other hand, the optimal scheduling algorithm was advantageous in terms of SOC management. The maximum-capacity bidding plan did not manage the SOC, which can cause problems in the operation the next day; in contrast, the optimal scheduling algorithm did manage the SOC by using constraints Equations (9) and (10), which ensured stable operation every day.

\section{Real-Time State-of-Charge Management Algorithm}

In a real-time operation, the ESS performs frequency regulation in response to an AGC signal every two seconds. The charging/discharging efficiency of the ESS causes an SOC error whenever the ESS provides frequency regulation. Because the SOC error would prevent the ESS from providing frequency regulation, a novel $S O C$ management algorithm is proposed. In a real-time operation, the $S O C$ is calculated as follows:

$$
\begin{aligned}
& \text { If } A G C(z) \leq 0, \operatorname{SOC}(z+1)=\operatorname{SOC}(z)-\frac{c\left(x_{i}\right) \times\left[A G C(z)+b p\left(x_{i}\right)\right]}{C_{\text {rated }}} \times \eta_{c} \times \Delta t \\
& \text { If } A G C(z)>0, \operatorname{SOC}(z+1)=\operatorname{SOC}(z)-\frac{c\left(x_{i}\right) \times\left[A G C(z)+b p\left(x_{i}\right)\right]}{C_{\text {rated }}} \times \frac{1}{\eta_{d}} \times \Delta t
\end{aligned}
$$

where $A G C(z)$ is the actual AGC signal, and $z$ is the frequency regulation interval. To solve the SOC error, this study presents a real-time $S O C$ management algorithm. This algorithm changes the base point through hysteresis loop according to the $S O C$ range. Figure 4 illustrates the concept of a real-time $S O C$ management algorithm. When the $S O C$ is between $S O C_{\text {min_limit }}$ and $S O C_{\text {max_limit }}$, the base point is set to $b p\left(x_{i}\right)$. The ESS then operates according to the optimal scheduling algorithm. When the SOC is above $S O C_{\text {max }}$ limit because of the accumulation of $S O C$ errors, $b p\left(x_{i}+2\right)$ is set to $B P_{\text {dis }}$ by rebidding the base point after two hours' operation according to PJM market rules. Owing to the changing base point, the ESS provides as much additional active power in the energy market as $B P_{\text {dis }}$ until the $S O C$

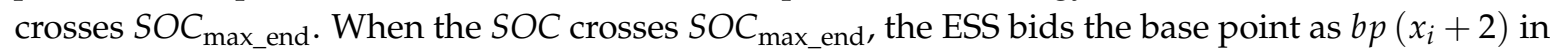
the optimal scheduling algorithm results. When the $S O C$ is below $S O C_{\min \_l i m i t}, b p\left(x_{i}+2\right)$ is set to $B P_{c h}$ by rebidding the base point, and the ESS provides as much additional active power in the energy

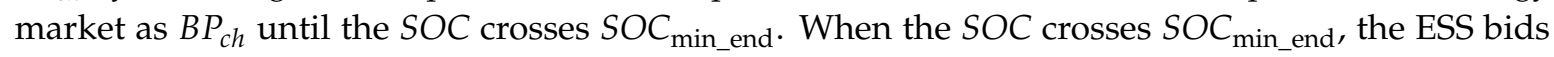
the base point as $b p\left(x_{i}+2\right)$ in the optimal scheduling algorithm results. Table 2 lists the parameters selected to maintain a stable $S O C$ range for real-time $S O C$ management. $S O C_{\min \_l i m i t}$ and $S O C_{\text {max_limit }}$ was set to $45 \%$ and $75 \%$, respectively. $S O C_{\text {min_end }}$ and $S O C_{\text {max_end }}$ was set to $50 \%$ and $70 \%$ respectively. The stable SOC range was set from $40 \%$ to $80 \%$. 


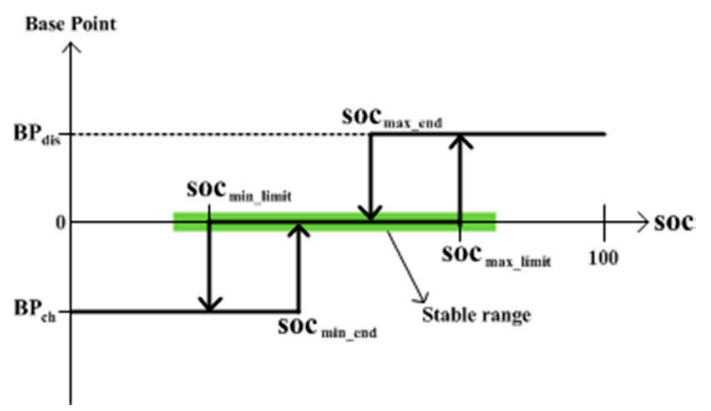

Figure 4. The concept of real-time SOC management algorithm.

Table 2. Simulation case results.

\begin{tabular}{ccccc}
\hline Case & Regulation Profit & Energy Profit & Total Profit & SOC \\
\hline Case 1 & $\$ 911.8$ & $\$ 0$ & $\$ 911.8$ & $10 \%$ \\
Case 2 & $\$ 1116.2$ & $\$-10.4$ & $\$ 1105.8$ & $10 \%$ \\
Case 3 $(0.05$ p.u) & $\$ 2630.3$ & $\$-147.4$ & $\$ 2482.9$ & $39.9 \%$ \\
Case 3 $(0.1$ p.u $)$ & $\$ 2555.5$ & $\$-159.7$ & $\$ 2395.8$ & $48.9 \%$ \\
Case 3 $(0.15$ p.u) & $\$ 2506.2$ & $\$-157.2$ & $\$ 2349$ & $37.4 \%$ \\
\hline
\end{tabular}

\section{Simulation Results}

This section reports the simulation results to evaluate the performance of the proposed algorithm using a real operation parameter. In the first case, the maximum-capacity bidding plan was performed, the second case used the optimal scheduling algorithm, and the third case applied the real-time SOC management algorithm to the optimal scheduling algorithm. The PSCAD/EMTDC (Manitoba HVDC Research Centre, Winnipeg, MB, Canada) was used to confirm the SOC changes using Equation (12) when the ESS performed frequency regulation in a real-time operation. The actual AGC signal data in the PJM on 5 September 2013 were used to calculate the SOC in real-time. Because the AGC cycle of the PJM is $2 \mathrm{~s}$, the $S O C$ was calculated every $2 \mathrm{~s}$. For the simulation, the scheduling was set as described in Section 4. To protect the ESS, the operational SOC range was set between $10 \%$ and $90 \%$. Therefore, the ESS will shut down to protect itself when the SOC exceeds the operational SOC range. Case 1 is result by maximum capacity bidding plan, case 2 is result by optimal scheduling algorithm and case 3 is result by optimal scheduling algorithm with real-time SOC management by various base point. Cases 1 and 2 were compared to evaluate the optimal scheduling algorithm, which was confirmed by the frequency regulation profit change and increased operation time. In addition, Case 2 was compared with Case 3 to evaluate the real-time SOC management algorithm by changing the base point. Table 2 shows total case results.

\subsection{Case 1}

Figure 5 shows the SOC changes when the ESS performed frequency regulation through the maximum-capacity bidding plan. In a real-time operation, the ESS performs frequency regulation by charging and discharging in response to an AGC signal. At 1 a.m., the SOC difference between the real-time operation SOC (55.8\%) and maximum-capacity bidding plan $(59.8 \%)$ was $4 \%$ because of the charging/discharging efficiency. This SOC difference increased consistently when the ESS performed the frequency regulation in a real-time operation. At 10 a.m., the SOC reached 10\%, and the ESS was shut down. When the ESS performed frequency regulation according to the maximum-capacity bidding plan, the performance score decreased because the ESS does not perform frequency regulation when the $S O C$ reaches $10 \%$. According to the decreasing performance score, the frequency regulation profit decreased from $\$ 2773.40$ to $\$ 911.80$. 


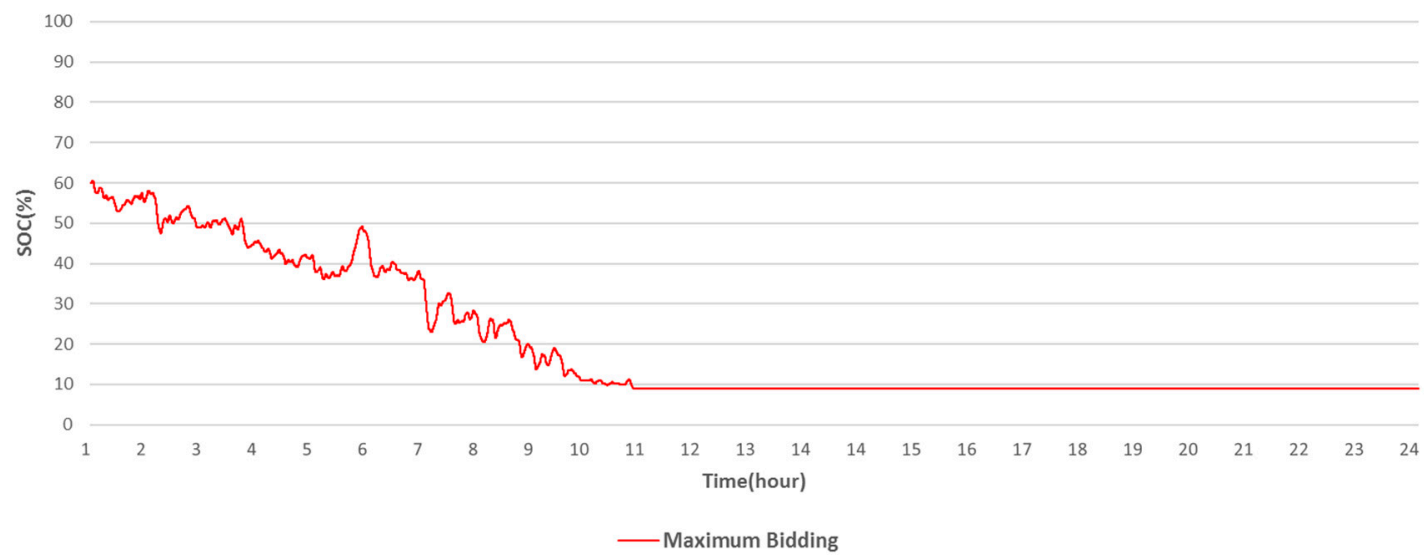

Figure 5. SOC of ESS in real-time operation simulation (Case 1-maximum bidding capacity plan).

\subsection{Case 2}

Figure 6 shows the SOC changes when the ESS performed frequency regulation through the optimal scheduling algorithm of Section 4 . The SOC decreased consistently when the ESS performed frequency regulation. According to the optimal scheduling algorithm, the base point of the ESS was set to -0.1 p.u for charging the SOC at 6 a.m. By changing the base point, the ESS recovered the SOC. Nevertheless, the real-time SOC reached $10 \%$ at 1 p.m., and the ESS was shut down. On the other hand, the simulation result of the optimal scheduling algorithm operated $3 \mathrm{~h}$ longer than the maximum-capacity bidding plan because the optimal scheduling algorithm managed the SOC by constraint in Equation (10). In the planning step, the maximum-capacity bidding plan earned more frequency regulation profit than the optimal scheduling algorithm (more than \$280.90); however, in the real-time operation, the optimal scheduling algorithm earned more frequency regulation profit than the maximum-capacity bidding plan (as much as \$194.00) through its scheduling capacity and base point. This result means that the optimal scheduling algorithm can increase the operation time of the ESS and earn more frequency regulation profit.

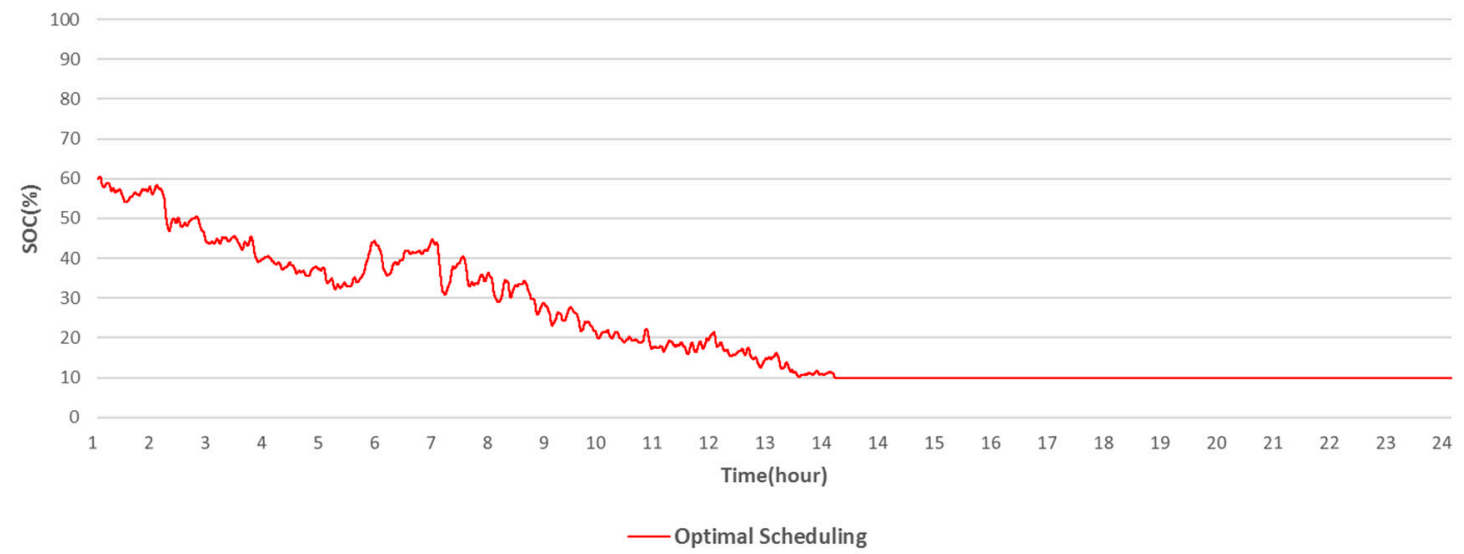

Figure 6. SOC of ESS in real-time operation simulation (Case 2-optimal scheduling algorithm).

\subsection{Case 3}

Figure 7 presents the SOC changes when the ESS performed frequency regulation through the optimal scheduling algorithm and real-time $S O C$ management algorithm by various base points. In the 0.05-p.u case, at 3 a.m., the SOC reached $43.7 \%$. Consequently, the bidding schedule of the base point was changed to -0.05 p.u through the real-time $S O C$ management algorithm after $2 \mathrm{~h}$ according to the PJM market rules until 9 a.m., when the SOC reaches more than $50 \%$. The bidding schedule can 
change $1 \mathrm{~h}$ before the operation time according to PJM market rules, thus, the bidding schedule was changed at 4 a.m. because the bidding schedule of 3 a.m. had already been submitted. Although the SOC reached more than $50 \%$ at 3 p.m., the bidding schedule was not changed until 4 p.m. for the same reason. Owing to the changed base point, the bidding capacity was also changed to $3.8 \mathrm{MW}$ by constraint Equation (12). In this case, the ESS maintained recovery time for $16 \mathrm{~h}$ because $0.05 \mathrm{p} . \mathrm{u}$ was not enough for SOC recovery. The SOC reached $39.9 \%$ when the ESS finished operating. The frequency regulation earned a profit of $\$ 2482.90$. In the 0.1-p.u case, the ESS maintained the recovery time for $8 \mathrm{~h}$, and the 0.1-p.u case recovery was more rapid and stable than that of the 0.05-p.u case. The SOC reached $48.9 \%$ in the stable range, and the frequency regulation earned a profit of $\$ 2395.80$. In the 0.15-p.u case, the ESS recovery was more rapid than that of the 0.1-p.u case; however, the 0.15-p.u case recovered too much because the $S O C$ reached $81.7 \%$ at 5 p.m. The SOC management algorithm changed the base point to 0.15 p.u, causing the $S O C$ to escape the stable range again. In this case, the ESS maintained the recovery time for $9 \mathrm{~h}$, and the SOC reached $37.4 \%$. The frequency regulation earned a profit of $\$ 2349$. The ESS of Case 3 used the optimal scheduling algorithm and real-time SOC management algorithm to perform frequency regulation for a day. In contrast, Case 2, which used only the optimal scheduling algorithm, did not perform for a day. This result shows that when an ESS performs frequency regulation without an additional SOC management algorithm, the ESS does not perform for a day, and the proposed SOC management algorithm successfully managed the SOC of the ESS. Moreover, the base point is a significant factor in this algorithm because the base point determines the recovery quantity.

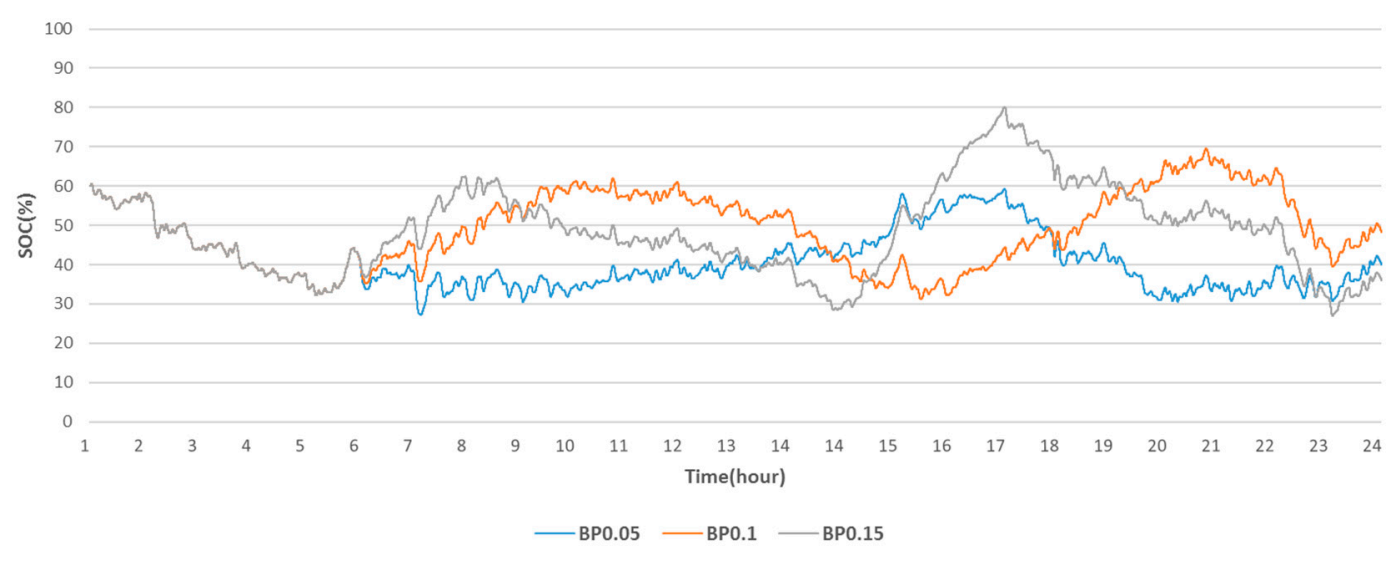

Figure 7. SOC of ESS in real-time operation simulation (Case 3-optimal scheduling algorithm with real-time SOC management).

Table 3 lists simulation results for different day. As shown Table 3, the base point is an important factor in this algorithm because the base point determines the recovery quantity of the SOC. Furthermore, for the determination base point, real-time SOC management algorithm should use accurate forecasting information. 
Table 3. Additional simulation case results.

\begin{tabular}{cccc}
\hline $\begin{array}{c}\text { Optimal Scheduling \& } \\
\text { Real-Time SOC Managment }\end{array}$ & Base Point & Profit & SOC \\
\hline \multirow{2}{*}{2013.06 .10} & 0.05 & $\$ 1254.2 \rightarrow \$ 211.7$ & $10 \%$ \\
& 0.1 & $\$ 1254.2 \rightarrow \$ 948.4$ & $25.61 \%$ \\
2013.12 .2 & 0.15 & $\$ 1254.2 \rightarrow \$ 953.6$ & $40.60 \%$ \\
\hline \multirow{2}{*}{2014.03 .10} & 0.05 & $\$ 2232.4 \rightarrow \$ 1857.1$ & $51.42 \%$ \\
& 0.1 & $\$ 2232.4 \rightarrow \$ 1815.1$ & $37.89 \%$ \\
& 0.15 & $\$ 2232.4 \rightarrow \$ 1778.1$ & $42.32 \%$ \\
\hline & 0.05 & $\$ 4024.3 \rightarrow \$ 3536.8$ & $52.12 \%$ \\
& 0.1 & $\$ 4024.3 \rightarrow \$ 3301.4$ & $65.56 \%$ \\
& 0.15 & $\$ 4024.3 \rightarrow \$ 3475.8$ & $41.21 \%$ \\
\hline
\end{tabular}

\section{Conclusions}

This study proposed an optimal scheduling algorithm for frequency regulation by an ESS to ensure the realization of maximum profit within a stable $S O C$ range. The maximum-capacity bidding plan is the best method for realizing frequency regulation profit, but it does not manage the SOC. The optimal scheduling algorithm schedules a bidding plan using the PSO method to realize the maximum frequency regulation profit within a stable $S O C$ range. Although the optimal scheduling algorithm provides less frequency regulation profit than the maximum-capacity bidding plan, the former maintains stable operation through SOC management. The proposed algorithm was evaluated through simulation of three cases by means of the PSCAD/EMTDC tool in a real-time operation. The simulation results show that the optimal scheduling algorithm increases the duration of real-time operation and results in higher profits in comparison to the conventional bidding plan without SOC management. While the conventional maximum-capacity bidding plan provides more profit than the optimal scheduling algorithm in the planning step, the optimal scheduling algorithm provides more profit in real-time operations.

This study also proposed a real-time SOC management algorithm. The ESS constantly performs frequency regulation using this algorithm by which the ESS changes the base point to recover the SOC in a real-time operation. In the case where the real-time $S O C$ management algorithm was applied in the PJM regulation market, it effectively recovered the $S O C$ when the base point was changed to 0.1 p.u. The proposed real-time $S O C$ management algorithm can be applied to various regulation market environments by changing the base point. This algorithm is expected to promote the participation of ESSs in various regulation markets.

Future work will deal with the forecasting error. The cases in this study were simulated using actual operation data, but the ESS uses forecast data during actual operation. Accordingly, the proposed algorithm should consider ESS potential forecasting error.

Acknowledgments: This research was supported by “Development of Operational criteria and Market trading procedure for Regulation Service using Battery Energy Storage" funded by Ministry of Trade, Industry \& Energy of Korea (grant number: 1004101337X1); This work was supported by the Power Generation \& Electricity Delivery Core Technology Program of the Korea Institute of Energy Technology Evaluation and Planning (KETEP), granted financial resource from the Ministry of Trade, Industry \& Energy, Republic of Korea (No. 20142010103010); This research was supported by Basic Science Research Program through the National Research Foundation of Korea (NRF), funded by the Ministry of Science, ICT \& Future Planning (grant number: NRF-2013R1A1A1012667).

Author Contributions: Jin-Sun Yang is lead author. Jin-Young Choi and Dong-Jun Won provided guidance. Geon-Ho An, Young-Jun Choi and Myoung-Hoe Kim provided Industrial research support.

Conflicts of Interest: The authors declare no conflict of interest. 


\section{References}

1. Wu, D.; Tang, F.; Dragicevic, T.; Juan, C.; Vasquez, J.; Guerrero, J. A control architecture to coordinate renewable energy sources and energy storage systems in islanded microgrids. IEEE Trans. Smart Grid 2015, 6, 1156-1166. [CrossRef]

2. Karami, H.; Sanjari, M.; Hosseinian, S.; Gharehpetian, G. An optimal dispatch algorithm for managing residential distributed energy resources. IEEE Trans. Smart Grid 2014, 5, 2360-2367. [CrossRef]

3. Erdinc, O.; Paterakis, N.; Mendes, T.; Bakirtzis, A.; Catalão, J. Smart household operation considering bi-directional EV and ESS utilization by real-time pricing-based DR. IEEE Trans. Smart Grid 2015, 6, 1281-1291. [CrossRef]

4. Jiang, B.; Fei, Y. Smart Home in Smart Microgrid: A cost-effective energy ecosystem with intelligent hierarchical agents. IEEE Trans. Smart Grid 2015, 6, 3-13. [CrossRef]

5. Tran, D.; Khambadkone, A. Energy management for lifetime extension of energy storage system in micro-grid applications. IEEE Trans. Smart Grid 2013, 4, 1289-1296. [CrossRef]

6. Daneshi, H.; Srivastava, A. Security-constrained unit commitment with wind generation and compressed air energy storage. IET Gener. Transm. Distrib. 2012, 6, 167-175. [CrossRef]

7. Su, H.; Gamal, A. Modeling and analysis of the role of energy storage for renewable integration: Power balancing. IEEE Trans. Power Syst. 2013, 4, 4109-4117. [CrossRef]

8. Rahbar, K.; Xu, J.; Zhang, R. Real-time energy storage management for renewable integration in microgrid: An off-line optimization approach. IEEE Trans. Smart Grid 2015, 6, 124-134. [CrossRef]

9. Wang, P.; Gao, Z.; Tjernberg, L. Operational adequacy studies of power systems with wind farms and energy storages. IEEE Trans. Power Syst. 2013, 27, 2377-2384. [CrossRef]

10. Zhang, L.; Li, Y. Optimal Energy management of wind-battery hybrid power system with two-scale dynamic programming. IEEE Trans. Sustain. Energy 2013, 4, 765-773. [CrossRef]

11. Wu, D.; Tang, F.; Dragicevic, T.; Vasquez, J.; Guerrero, J. Autonomous active power control for islanded AC microgrids with photovoltaic generation and energy storage system. IEEE Trans. Energy Convers. 2014, 29, 882-892. [CrossRef]

12. Damiano, A.; Gatto, G.; Marongiu, I.; Porru, M.; Serpi, A. Real-time control strategy of energy storage systems for renewable energy sources exploitation. IEEE Trans. Sustain. Energy 2014, 5, 567-576. [CrossRef]

13. Shiltz, D.; Cvetkovi'c, M.; Annaswamy, A. An integrated dynamic market mechanism for real-time markets and frequency regulation. IEEE Trans. Sustain. Energy 2016, 7, 875-885. [CrossRef]

14. Choi, J.; Heo, S.; Kim, M. Hybrid operation strategy of wind energy storage system for power grid frequency regulation. IET Gener. Transm. Distrib. 2016, 10, 736-749. [CrossRef]

15. Dang, J.; Seuss, J.; Suneja, L.; Harley, R. SOC feedback control for wind and ESS hybrid power system frequency regulation. IEEE J. Emerg. Sel. Top. Power Electron. 2014, 2, 79-86. [CrossRef]

16. Papalexopoulos, A.; Andrianesis, P. Performance-based pricing of frequency regulation in electricity markets. IEEE Trans. Power Syst. 2014, 29, 441-449. [CrossRef]

17. He, G.; Chen, Q.; Kang, C.; Pinson, P.; Xia, Q. Optimal bidding strategy of battery storage in power markets considering performance-based regulation and battery cycle life. IEEE Trans. Smart Grid 2015, 7, $2359-2367$. [CrossRef]

18. Singh, H.; Papalexopoulos, A. Competitive procurement of ancillary services by an independent system operator. IEEE Trans. Power Syst. 1999, 14, 498-504. [CrossRef]

19. Papalexopoulos, A.; Singh, H. On the various design options for ancillary services markets. In Proceedings of the 34th Hawaii International Conference on System Sciences (HICSS-34), Maui, HI, USA, 3-5 January 2001.

20. PJM Manual 11: Energy \& Ancillary Services Market Operations. Available online: http://www.pjm.com/ / media/documents/manuals/m11.ashx (accessed on 9 April 2015).

21. Preliminary Billing Data. Available online: http://www.pjm.com/pub/account/pjm-regulation-data/ 201309.csv (accessed on 5 September 2013).

22. Market \& Operation, Market-Based Regulation. Available online: http://www.pjm.com/ /media/marketsops/ancillary/mkt-based-regulation/regulation-data.ashx (accessed on 13 August 2014).

23. Hourly Real-Time \& Day-Ahead LMP. Available online: http://www.pjm.com/pub/account/lmpmonthly/ 201309-rt.csv (accessed on 2 January 2014). 
24. Li, S.; Zhang, D.; Roget, A.; O'Neill, Z. Integrating home energy simulation and dynamic electricity price for demand response study. IEEE Trans. Smart Grid 2014, 5, 779-788. [CrossRef]

25. Faria, P.; Soares, J.; Vale, Z.; Morais, H.; Sousa, T. Modified particle swarm optimization applied to integrated demand response and DG resources scheduling. IEEE Trans. Smart Grid 2013, 4, 606-616. [CrossRef]

26. Nikmehr, N.; Ravadanegh, S. Optimal power dispatch of multi-microgrids at future smart distribution grids. IEEE Trans. Smart Grid 2015, 6, 1648-1657. [CrossRef]

27. Basu, A.; Bhattacharya, A.; Chowdhury, S.; Chowdhury, S. Planned scheduling for economic power sharing in a CHP-based micro-grid. IEEE Trans. Power Syst. 2012, 27, 30-38. [CrossRef]

(C) 2016 by the authors; licensee MDPI, Basel, Switzerland. This article is an open access article distributed under the terms and conditions of the Creative Commons Attribution (CC-BY) license (http:/ / creativecommons.org/licenses/by/4.0/). 